1 Doutora em Enfermagem pela Universidade de São Paulo (USP) - São Paulo (SP). Professora adjunta da Universidade Federal de Alfenas (Unifal) - Alfenas (MG), Brasil.

simonealbino76@hotmail.com

2 Livre docente pela Universidade de São Paulo (USP) - São Paulo (SP).

Professora da Universidade de São Paulo (USP) - São Paulo (SP).

lislaine@usp.br

\section{Avaliação da Estratégia Saúde da Família: perspectiva dos usuários em Minas Gerais, Brasil}

\author{
The Family Health Strategy assessment: perspective of users in Minas \\ Gerais, Brazil
}

Simone Albino da Silva', Lislaine Aparecida Fracolli2
RESUMO A Estratégia Saúde da Família (ESF) tem papel fundamental na Atenção Primária à Saúde no Brasil. Avaliou-se a ESF na visão do usuário, numa região de saúde de Minas Gerais, por meio de pesquisa avaliativa, quantitativa e transversal, utilizando o Primary Care Assessment Tool (PCATool). Os usuários têm na ESF o principal recurso de atenção à saúde e a valorizam como coordenadora do cuidado nos demais níveis assistenciais. Contudo, consideram que existem barreiras organizacionais no acesso; as necessidades da comunidade não são a base para a oferta dos serviços; na organização das ações não há espaço para a participação do usuário; e nem sempre a família é a unidade central do cuidado.

PALAVRAS-CHAVE Atenção Primária à Saúde; Avaliação de serviços de saúde; Estratégia Saúde da Família.

ABSTRACT The Family Health Strategy (ESF) has key role in Primary Health Care in Brazil. It has assessed the ESF in the user perspective, in a health region of Minas Gerais, by means of an evaluative, quantitative and cross-sectional survey, using the Primary Care Assessment Tool (PCATool). Users have the ESF as the main resource of health care and have valued it as the coordinator of care in other healthcare levels. However, they consider that there are organizational barriers in access; community needs are not the basis to the range of services; in the organization of the actions there is no place for the user participation, and the family is not always the central unit of care.

KEYWORDS Primary Health Care; Health service evaluation; Family Health Strategy. 


\section{Introdução}

A Estratégia Saúde da Família (ESF) e o Programa de Agentes Comunitários de Saúde (Pacs) foram os arranjos estruturais adotados pelo governo brasileiro, a partir de meados dos anos 1990, para reorientar o modelo de atenção à saúde por meio de práticas de saúde multiprofissionais. Dessa forma, foi possível organizar e expandir a rede de serviços e de ações básicas de saúde no nível municipal (GIL, 2006).

A mudança do modelo de atenção à saúde apoiou-se nos resultados das discussões da Conferência Mundial de Cuidados Primários de Saúde de Alma Ata e nos princípios de conquista do direito à saúde, de justiça social e de cidadania, constantes do ideário da reforma sanitária brasileira, consolidados na VIII Conferência Nacional de Saúde e na criação do Sistema Único de Saúde (SUS). Logo, a implementação da ESF aconteceu claramente como uma maneira de viabilizar e fortalecer os princípios do SUS (ANDRADE; BUENO; BEZERRA, 2006).

No início dos anos 2000, com respaldo legal, administrativo e financeiro, iniciou-se uma nova fase de ampliação da cobertura com a ESF. Esse movimento sofreu influências políticas nacionais e internacionais, filosóficas, empíricas e científicas, como resultados de estudos da temática da Atenção Primária à Saúde (APS), alavancados, principalmente, por Starfield (2002), que, além de reunir publicações que comprovam a eficiência de sistemas de saúde baseados na APS, também organizou o conjunto de atributos próprios desse nível assistencial: acesso de primeiro contato, longitudinalidade, integralidade e coordenação (atributos essenciais) e os chamados atributos derivados: orientação familiar, orientação comunitária e competência cultural.

$\mathrm{Na}$ busca bibliográfica realizada para a composição deste trabalho, verificou-se que, no Brasil, a publicação de pesquisas de avaliação sobre a atuação da APS, que não estejam relacionadas a uma doença ou um agravo específico, é incipiente. Em sua maioria, são avaliações do impacto da implantação da ESF sobre a saúde e o bem-estar da população por meio de indicadores fortemente determinados por fatores como renda, saneamento, educação, entre outros (MACINKO; ALMEIDA; OLIVEIRA, 2003). Considerou-se, também, que grande parte dos estudos de avaliação da APS, publicados nos últimos 14 anos, foi fomentada pelo governo federal brasileiro. A maioria desses estudos foi realizada em grandes municípios, na busca de subsídios científicos que ratificassem a continuidade e a expansão da ESF (BODSTEIN, ET AL., 2006).

Diante da magnitude da ESF no sistema de saúde brasileiro e do contexto científico exposto, justifica-se a realização de novos estudos avaliativos, em diferentes regiões do País, em municípios de portes variados, com o uso de diversas propostas avaliativas e metodológicas.

A concepção adotada para este estudo é a da avaliação da assistência ao usuário no primeiro nível de atenção, abarcando as dimensões da APS como a conquista do direito à saúde integral, como o ponto central da organização de uma política e de um sistema de saúde e como dimensão tecnológica de implementação dos atributos essenciais e derivados propostos por Starfield (2002).

O objetivo deste trabalho foi avaliar a ESF na perspectiva dos usuários, buscando-se identificar se os atributos de acesso de primeiro contato, de integralidade, de coordenação da atenção, de longitudinalidade, de orientação familiar e de orientação comunitária estão incorporados às práticas das equipes de saúde.

\section{Metodologia}

Trata-se de uma pesquisa avaliativa (NOVAES, 2000), de abordagem quantitativa, com delineamento transversal (POLIT; BECK; HUNGLER, 
2004), derivada da pesquisa Avaliação dos Atributos da Atenção Primária à Saúde na Estratégia Saúde da Família em Municípios do Sul de Minas Gerais, aprovada pelo comitê de ética da Escola de Enfermagem da Universidade de São Paulo - EEUSP, parecer 30699, de 01/06/2012.

A amostragem dos participantes foi realizada pelo método de cotas (GIL, 1999), com estimativa da média com desvio padrão de 1,2 , erro de 0,1 e intervalo de confiança de $95 \%$. O número total de participantes foi dividido proporcionalmente entre as cidades e o tamanho da população coberta pela ESF.

Os participantes foram pessoas maiores de 18 anos, residentes e cadastradas há mais de um ano em área de abrangência de unidades da ESF de zona urbana, que funcionassem ininterruptamente há pelo menos cinco anos, em municípios da região de saúde de Alfenas, com cobertura populacional pela ESF superior a $50 \%$. Todos os participantes assinaram o Termo de Consentimento Livre e Esclarecido, em conformidade com a legislação vigente.

Os dados foram coletados nos meses de junho e julho de 2012, por meio de entrevistas feitas no domicílio, com duração média de quarenta minutos. Utilizou-se como roteiro de entrevista o PCATool, que é um instrumento elaborado com base nos pressupostos de estrutura, de processo e de resultado, que compõem a avaliação em saúde proposta por Donabedian (DONABEDIAN, 2005). Esse instrumento foi desenvolvido nos EUA por Starfield (2002) e vem sendo traduzido e validado em diferentes países, inclusive no Brasil (BRASIL, 2010).

O PCATool, na versão dirigida ao usuário adulto, possui 88 questões. As três perguntas iniciais visam a identificar qual unidade de saúde o usuário tem como referência de atendimento e o grau de afiliação a esse serviço. Esse item é pontuado de 1 a 4 e não se constitui num atributo. Na sequência, há 85 perguntas distribuídas entre os atributos essenciais da APS: Acesso de Primeiro
Contato - Utilização; Acesso de Primeiro Contato - Acessibilidade; Longitudinalidade; Coordenação - Integração de Cuidados; Coordenação - Sistema de Informações; Integralidade - Serviços Disponíveis; Integralidade - Serviços Prestados; e entre os atributos derivados de Orientação Familiar e Orientação Comunitária. As respostas possíveis para cada item são: 'com certeza sim' (valor $=4$ ); 'provavelmente sim' (valor=3); 'provavelmente não' (valor $=2$ ); 'com certeza não' (valor=1); e 'não sei/não lembro' (valor $=9$ ). Os escores para cada um dos atributos ou seus componentes são calculados pela média aritmética simples dos valores das respostas dos itens que os compõem.

Como resultado geral da avaliação pelo PCATool, tem-se duas medidas: o Escore Essencial, que é a média dos escores dos componentes que pertencem aos atributos essenciais, e o Escore Geral, que é a média dos escores dos componentes dos atributos essenciais, acrescida dos escores dos atributos derivados. Esses resultados caracterizam o grau de orientação do serviço ou do sistema de saúde aos atributos da Atenção Primária à Saúde.

As respostas foram organizadas em um banco de dados criado no software Microsoft Excel for Windows, e a análise estatística foi realizada no software Statistical Package for the Social Sciences - SPSS 14.0, com intervalo de confiança de $95 \%$. Para a aferição de cada atributo, foi feito um escore, utilizando-se a média aritmética das respostas e este é comparado ao valor de referência 6,66 , limite entre alto e baixo escore. Para a comparação dos escores médios dos atributos da APS por grupos, utilizou-se o modelo Anova, com post hoc, pelo método Tukey. Cada atributo também foi analisado considerando-se cada item, tendo como referência o percentual de $66,6 \%$ adotado no PCATool. Essa análise teve a consistência interna e a confiabilidade avaliadas pelo método Alfa de Cronbach (POLIT; BECK; HUNGLER, 2004). 


\section{Resultados e discussão}

A região de saúde de Alfenas, localizada no Estado de Minas Gerais, é composta por 17 municípios, sendo: seis com menos de dez mil habitantes; cinco com até 20 mil habitantes; cinco com até 40 mil habitantes; e um com mais de 50 mil habitantes. Atenderam aos critérios de inclusão 33 unidades de saúde da ESF (localizadas em 11 municípios diferentes), em cuja área de abrangência foi possível entrevistar 527 adultos. Os participantes têm como características serem do sexo feminino (86,15\%); casados $(59,96 \%)$; de cor branca $(62,81 \%)$; com até três filhos (50,10\%); com idade média de 53,36 anos para as mulheres e de 58,23 para os homens. O valor do grau de afiliação e os escores médios dos atributos da APS, conferidos pelos participantes, estão expostos na tabela 1.

Tabela 1. Valores médios e respectivos desvios-padrão dos atributos da Atenção Primária à Saúde, Escore Essencial e Escore Geral, conferidos pelos usuários adultos na avaliação da Estratégia Saúde da Família, microrregião de Alfenas MG, 2012

\begin{tabular}{lrrrrr}
\hline Atributo da APS & N & Mínimo & Máximo & Média & DP \\
\hline Grau de Afiliação & 527 & 1,00 & 4,00 & 3,67 & 0,62 \\
Acesso de Primeiro Contato - Utilização & 527 & 0,00 & 10,00 & 8,59 & 1,97 \\
Acesso de Primeiro Contato - Acessibilidade & 524 & 0,00 & 7,50 & 3,21 & 1,49 \\
Longitudinalidade & 527 & 0,71 & 10,00 & 7,26 & 1,96 \\
Coordenação - Integração de Cuidados & 259 & 0,00 & 10,00 & 6,10 & 2,67 \\
Coordenação - Sistema de Informação & 510 & 0,00 & 10,00 & 6,41 & 2,16 \\
Integralidade - Serviços Disponíveis & 460 & 0,45 & 9,24 & 5,22 & 1,56 \\
Integralidade - Serviços Prestados & 508 & 0,00 & 10,00 & 4,92 & 2,50 \\
Orientação Familiar & 511 & 0,00 & 10,00 & 5,69 & 2,93 \\
Orientação Comunitária & 464 & 0,00 & 10,00 & 5,88 & 2,48 \\
Escore Essencial & 527 & 2,37 & 8,43 & 5,64 & 1,09 \\
Escore Geral & 527 & 2,21 & 8,75 & 5,67 & 1,20 \\
\hline
\end{tabular}

Fonte: Elaboração própria

Nos escores médios dos atributos da ESF não foi encontrada diferença estatística, comparando-se os grupos por sexo, idade, cor e presença de filhos. A comparação dos escores médios dos atributos entre os grupos classificados segundo o estado civil mostrou que o Acesso de Primeiro Contato - Acessibilidade é mais bem avaliado pelos participantes solteiros, casados/amasiados e viúvos do que pelos participantes divorciados.
O valor médio do grau de afiliação conferido pelo usuário da ESF da região de saúde de Alfenas foi alto, informando que o mesmo prioriza utilizar e depositar a responsabilidade pelo seu atendimento na ESF.

A busca do serviço é medida pelo atributo Acesso de Primeiro Contato - Utilização, que recebeu o mais alto escore médio, corroborando o resultado do grau de afiliação. Estudos em diferentes partes do Brasil mostraram que os usuários da ESF também 
avaliaram este atributo com altos escores (IBAÑEZ ET AL., 2006; MACINKO; ALMEIDA; SÁ, 2007; OLIVEIRA, 2007; OLIVEIRA ET AL., 2013; PEREIRA ET AL., 2011; REIS ET AL., 2013; SALA ET AL., 2011; VAN STRALEN ET AL., 2008; VIANA ET AL., 2008; VIANA, 2012).

Giovanella e Mendonça (2003) sugerem que, nas localidades onde ocorreu a substituição de rede básica tradicional pela ESF, há maior probabilidade de esta se constituir em uma fonte habitual de atenção. Na região de saúde em estudo, a ESF foi a escolha dos gestores para a ampliação da rede de serviços de atenção primária, com alta cobertura populacional nos municípios pesquisados, tornando-a um serviço de procura regular pela população.
Por outro lado, o atributo Acesso de Primeiro Contato - Acessibilidade, que avalia quanto o serviço de saúde é disponível ao usuário e sua capacidade de atendimento para a rotina ou para a demanda espontânea, recebeu a pior avaliação entre os demais. A falta dessa qualidade não é uma característica apenas dos municípios deste estudo, pois recebeu baixos escores dos usuários em outras avaliações da ESF (ELIAS ET AL., 2006; IBAÑEZ ET AL., 2006; MACINKO; ALMEIDA; DE SÁ, 2007; OLIVEIRA, 2007; OLIVEIRA ET AL., 2013; PEREIRA ET AL., 2011; REIS ET AL., 2013; SALA ET AL., 2011; VAN STRALEN ET AL., 2008; VIANA ET AL., 2008; VIANA, 2012). No detalhamento dos itens que compõe este atributo, expostos na tabela 2, pode-se compreender melhor esse resultado.

Tabela 2. Distribuição percentual das respostas dos usuários adultos da Estratégia Saúde da Família aos itens que compõem o atributo Acesso de Primeiro Contato - Acessibilidade, microrregião de Alfenas - MG, 2012. Alfa de Cronbach = 0,683, N = 524

\begin{tabular}{lrrrrrrrrrrrr}
\hline \multirow{2}{*}{ Avaliação } & $\mathbf{C 1}$ & $\mathbf{C 2}$ & $\mathbf{C 3}$ & $\mathbf{C 4}$ & $\mathbf{C 6}$ & $\mathbf{C 7}$ & $\mathbf{C 8}$ & $\mathbf{C 9}$ & $\mathbf{C 1 0}$ & $\mathbf{C 1 1}$ & $\mathbf{C 1 2}$ \\
\cline { 2 - 10 } & $\%$ & $\%$ & $\%$ & $\%$ & $\%$ & $\%$ & $\%$ & $\%$ & $\%$ & $\%$ & $\%$ \\
\hline Com certeza, sim & 0,19 & 0,76 & 20,46 & 25,10 & 1,72 & 0,95 & 43,30 & 10,67 & 38,93 & 32,89 & 50,38 \\
Provavelmente sim & 0,19 & 0,76 & 21,99 & 14,69 & 1,53 & 0,38 & 27,20 & 9,54 & 24,43 & 23,71 & 9,73 \\
Provavelmente não/não & 5,53 & 7,44 & 22,94 & 25,76 & 6,30 & 6,87 & 11,88 & 21,95 & 17,75 & 19,50 & 14,89 \\
sei não lembro & & & & & & & & & & & \\
Com certeza, não & 94,08 & 91,03 & 34,61 & 34,35 & 90,46 & 91,79 & 17,62 & 57,82 & 18,89 & 23,90 & 25,00 \\
Total & 100 & 100 & 100 & 100 & 100 & 100 & 100 & 100 & 100 & 100 & 100 \\
\hline
\end{tabular}

Fonte: Elaboração própria

Notas: C1 - O 'nome do serviço de saúde/ou nome médico/enfermeiro' fica aberto no sábado ou no domingo? C2 - O 'nome do serviço de saúde/ou nome médico/ enfermeiro' fica aberto pelo menos algumas noites de dias úteis até as 20 horas? C3 - Quando o seu 'nome do serviço de saúde/ou nome médico/enfermeiro' está aberto e você adoece, alguém de lá atende você no mesmo dia? C4 - Quando o seu 'nome do serviço de saúde/ou nome médico/enfermeiro' está aberto, você consegue aconselhamento rápido pelo telefone se precisar? C5 - Quando o seu 'nome do serviço de saúde/ou nome médico/enfermeiro' está fechado, existe um número de telefone para o qual você possa ligar quando fica doente? C6 - Quando o seu 'nome do serviço de saúde/ou nome médico/enfermeiro' está fechado no sábado e domingo e você fica doente, alguém deste serviço atende você no mesmo dia? C7 - Quando o seu 'nome do serviço de saúde/ou nome médico/enfermeiro' está fechado e você fica doente durante a noite, alguém deste serviço atende você naquela noite? C8 - É fácil marcar hora para uma consulta de revisão (consulta de rotina, 'check-up') neste 'nome do serviço de saúde/ou nome médico/enfermeiro?' C9 - Quando você chega ao seu 'nome do serviço de saúde/ou nome médico/enfermeiro', você tem que esperar mais de 30 minutos para se consultar com o médico ou enfermeiro (sem contar triagem ou acolhimento)? C10 - Você tem que esperar por muito tempo ou falar com muitas pessoas para marcar hora no seu 'nome do serviço de saúde/ou nome médico/enfermeiro?' C11 - É difícil para você conseguir atendimento médico do seu 'nome do serviço de saúde/ ou nome médico/enfermeiro' quando pensa que é necessário? C12 - Quando você tem que ir ao 'nome do médico/enfermeira/ local', você tem que faltar ao trabalho ou à escola para ir ao serviço de saúde?

O horário de atendimento e os meios de comunicação com o serviço obtiveram avaliações médias inferiores ao valor de classificação. Outros estudos também apontaram como obstáculos ao acesso a indisponibilidade de atendimento nos finais de semana (PEREIRA ET AL., 2011; REIS ET AL., 2013; SALA ET AL., 2011; VIANA, 2012) e após as 18 horas (REIS ET 
AL., 2013; SALA ET AL., 2011; VIANA, 2012), bem como a ausência de mecanismos de aconselhamento ou de agendamento por telefone no horário normal de funcionamento ou quando o serviço se encontra fechado (PEREIRA ET AL., 2011; REIS ET AL., 2013; VIANA, 2012). Para transpor esses obstáculos, é necessário que as pessoas faltem ao trabalho ou à escola para conseguir realizar agendamento ou atendimento. Em outros estudos, o horário estendido culminou em um resultado diferente, em que as pessoas não precisam mudar sua rotina para receber atendimento de saúde na ESF (REIS ET AL., 2013; SALA ET AL., 2011).

Existe dificuldade em agendar uma consulta para o mesmo dia nas unidades da ESF na microrregião de Alfenas, assim como em diversas outras cidades do País (PEREIRA ET AL., 2011; REIS ET AL., 2013; SALA ET AL., 2011; VIANA, 2012). Mesmo os usuários entrevistados avaliando que é necessário falar com muitas pessoas ou esperar muito tempo para conseguir um atendimento, julgaram ser fácil marcar uma consulta de rotina na ESF, (REIS ET AL., 2013; SALA ET AL., 2011; VIANA, 2012).

O tempo de espera para atendimentos agendados é menor que trinta minutos, o que não foi referido pelos usuários de outros trabalhos (PEREIRA ET AL., 2011; SALA ET AL., 2011; VIANA, 2012). Esse resultado expressa qualidade no planejamento do atendimento, pois é um importante parâmetro para medir a barreira de acessibilidade organizacional (STARFIELD, 2002), e exceder o limite de trinta minutos implica comprometimento do desempenho do serviço (PEREIRA ET AL., 2011).

Um aumento no número de barreiras ao acesso foi associado a menos atenção no primeiro contato e continuidade mais baixa, mesmo depois do controle de várias características influentes do paciente, tipos de centros de atenção primária e tipos de profissionais de atenção primária (STARFIELD, 2002).

$\mathrm{O}$ atributo Longitudinalidade recebeu o segundo maior escore médio na avaliação dos usuários, assim como em outros trabalhos (ELIAS ET AL., 2006; IBAÑEZ ET AL., 2006; MACINKO;
ALMEIDA; DE SÁ, 2007; OLIVEIRA ET AL., 2013; PEREIRA ET AL., 2011; REIS ET AL., 2013; SALA ET AL., 2011; VAN STRALEN ET AL., 2008; VIANA ET AL., 2008; VIANA, 2012).

Para Elias et al. (2006), o resultado positivo da avaliação da longitudinalidade é esperado, já que a ESF tem no seu ideário o estabelecimento de vínculo entre a família e os profissionais da unidade. Contudo, segundo Cunha e Giovanella (2011), a sua presença não é aquisição simples. Para esses autores, ainda que o estabelecimento do vínculo longitudinal esteja no âmbito da prática do profissional, o atendimento a tal atributo só é possível se for uma prioridade da gestão, na medida em que envolve questões como a oferta adequada de serviços de APS e os mecanismos de fixação do profissional na unidade de saúde.

Mesmo diante de um resultado positivo, alguns itens do atributo Longitudinalidade tiveram um resultado desfavorável. O item que versa sobre a possibilidade de se telefonar para o profissional de saúde em caso de dúvida apresentou avaliação negativa (38,78\%). A mesma barreira de comunicação já foi apontada no atributo Acesso de Primeiro Contato - Acessibilidade e também foi encontrada em outras avaliações (IBAÑEZ ET AL., 2006; VAN STRALEN ET AL., 2008; VIANA, 2012).

A avaliação do conhecimento do médico/ enfermeiro sobre as condições de vida do entrevistado, no que tange aos coabitantes por residência $(64,83 \%)$, aos problemas mais importantes $(65,84 \%)$ e às dificuldades para obtenção de medicamentos (61,45\%), apresentou resultados considerados baixos, apesar de próximos ao valor de referência. Os mesmos itens também tiveram avaliações semelhantes em outros estudos (PEREIRA ET AL., 2011; SALA ET AL., 2011; VIANA, 2012).

$O$ paradoxo entre as avaliações negativas nos itens acima e as avaliações positivas nos questionamentos se o médico/enfermeiro concede tempo suficiente para falar sobre as preocupações ou problemas (89\%) e se as perguntas dos usuários são respondidas de maneira compreensível (96,59\%) é questionado por Pereira et al. (2011), que encontraram 
resultados semelhantes em sua avaliação. Para esses autores, tal resultado mostra que o espaço destinado à escuta das necessidades do usuário pode se dar no sentido de abordagens voltadas para os aspectos biológicos, fato que pode ter contribuído para o usuário considerar o tempo da consulta suficiente.

O percentual de $62,05 \%$ dos entrevistados que responderam afirmativamente sobre a vontade de mudança do atual serviço/ médico/enfermeiro para outro serviço deixou evidente a insatisfação da maior parte dos usuários.

Os atributos Coordenação - Sistemas de Informação e Coordenação - Integração de Cuidados obtiveram valores considerados baixos, porém, próximos do valor limite de classificação de 6,66. Oliveira (2013), avaliando a ESF sob a ótica do idoso, também encontrou baixos escores médios, próximos do valor limite, para ambos os atributos. Nos estudos em que o atributo Coordenação não foi dividido, os usuários da ESF concederam altos escores médios (PEREIRA ET AL., 2011; SALA ET AL., 2011; VAN STRALEN ET AL., 2008; VIANA ET AL., 2008), e em um apresentou resultado desfavorável (IBAÑEZ ET AL., 2006; OLIVEIRA, 2007).
A questão inicial de avaliação do atributo Coordenação - Integração de Cuidados foi 'Você já foi consultar qualquer tipo de especialista ou serviço especializado no período em que você está em acompanhamento no nome do serviço de saúde/ou nome médico/ enfermeiro?'. Dos entrevistados, 48,81\% responderam sim, 49,80\% responderam não e $1,39 \%$ não souberam opinar. Somente os que responderam afirmativamente prosseguiram nas questões desse atributo.

Nota-se o elevado percentual de usuários a referir a utilização de serviços especializados na microrregião de Alfenas, resultado contrário aos achados de Sala et al. (2011). Esse percentual elevado é indicativo de baixa resolubilidade e qualidade da ESF. Almeida, Fausto e Giovanella (2011) elencam que, entre os fatores que indicam o fortalecimento da ESF como centro coordenador da assistência aos usuários, está a alta resolubilidade, de, no mínimo, 80\% dos atendimentos.

Os itens do atributo Coordenação Integração de Cuidados tratam da avaliação do usuário sobre a ESF como centro coordenador da assistência no sistema de saúde e estão expostos na tabela 3 .

Tabela 3. Distribuição percentual das respostas dos usuários adultos da Estratégia Saúde da Família aos itens que compõem o atributo Coordenação Integração de Cuidados, microrregião de Alfenas - MG, 2012. Alfa de Cronbach = 0,782, N= 259

\begin{tabular}{lrrrrrrrrr}
\hline \multirow{2}{*}{ Avaliação } & E2 & E3 & E4 & E5 & E6 & E7 & E8 & E9 \\
\cline { 2 - 8 } & & $\%$ & $\%$ & $\%$ & $\%$ & $\%$ & $\%$ & $\%$ & $\%$ \\
\hline Com certeza, sim & 73,46 & 86,87 & 28,19 & 58,37 & 44,79 & 55,60 & 40,93 & 35,91 \\
Provavelmente sim & 5,00 & 5,02 & 5,79 & 10,12 & 12,36 & 8,88 & 8,11 & 6,18 \\
Provavelmente não/não sei/não lembro & 4,23 & 2,70 & 13,90 & 2,72 & 9,27 & 10,04 & 13,13 & 13,90 \\
Com certeza, não & 17,31 & 5,41 & 52,12 & 28,79 & 33,59 & 25,48 & 37,84 & 44,02 \\
Total & 100 & 100 & 100 & 100 & 100 & 100 & 100 & 100 \\
\hline
\end{tabular}

Fonte: Elaboração própria

Notas: E2 - O 'nome do serviço de saúde/ou nome médico/enfermeiro' sugeriu (indicou, encaminhou) que você fosse se consultar com este especialista ou serviço especializado? E3 - O 'nome do serviço de saúde/ou nome médico/enfermeiro' sabe que você fez essas consultas com este especialista ou serviço especializado? E4 - O seu 'médico/enfermeiro' discutiu com você diferentes serviços onde você poderia ser atendido para este problema de saúde? E5 - O seu 'médico/enfermeiro' ou alguém que trabalha no/com 'nome do serviço de saúde' ajudou-o/a marcar esta consulta? E6 - O seu 'médico/enfermeiro' escreveu alguma informação para o especialista, a respeito do motivo desta consulta? E7 - O 'nome do serviço de saúde/ou nome médico/enfermeiro' sabe quais foram os resultados desta consulta? E8 - Depois que você foi a este especialista ou serviço especializado, o seu 'médico/enfermeiro' conversou com você sobre o que aconteceu durante esta consulta? E9 - O seu 'médico/enfermeiro' pareceu interessado na qualidade do cuidado que Ihe foi dado (Ihe perguntou se você foi bem ou mal atendido por este especialista ou serviço especializado)? 
As avaliações positivas confirmam a ESF como porta de entrada para os serviços especializados e também falam a favor da organização do sistema regional de saúde.

Os itens que tratam do fluxo da informação para a continuidade do cuidado e da qualidade deste em outros níveis assistenciais receberam avaliações negativas. Compondo um paralelo com outras avaliações de mesma metodologia, realizadas em cidades de grande porte populacional, a maioria dos itens tratados no início do parágrafo não obteve um resultado satisfatório (PEREIRA ET AL., 2011; SALA ET AL., 2011; VIANA, 2012). A partir disso, é possível considerar que esse resultado não é prerrogativa dos pequenos municípios pela estrutura de saúde existente, mas pela não valorização da opinião do usuário, pela indisponibilidade da referência e contrarreferência e pela comunicação ineficiente entre usuário e profissional.

A essência da coordenação é a disponibilidade de informações a respeito de problemas e serviços anteriores e o reconhecimento daquela informação, na medida em que está relacionada às necessidades para o presente atendimento. (STARFIELD, 2002, P.365-366).

Cabe ressaltar que a ESF tem o propósito de se conformar como porta de entrada e como filtro do fluxo para o sistema de saúde, de ter no profissional a figura de coordenador dos cuidados ao usuário, conduzindo-o pela rede assistencial na busca da melhor qualidade assistencial possível. Para isso, necessita-se estabelecer e fortalecer o fluxo de informação na rede de serviços (GIOVANELLA; ESCOREL; MENDONÇA, 2003).

Para Ibanez et al. (2006), o atributo Coordenação não faz parte das dimensões historicamente valorizadas nos serviços de saúde, não compondo o aprendizado que a população construiu nas suas relações com os serviços, embora a coordenação seja um dos pilares da concepção estruturante e complexa da atenção primária em um sistema de saúde. Transformar essa experiência vivida pelo usuário requer investimento forte e duradouro, não somente em capacitação, mas num esforço de mudança cultural de profissionais, de gestores e de sustentabilidade das propostas implantadas.

O atributo Coordenação - Sistema de Informação obteve baixo escore, contudo, o resultado ficou próximo ao valor limite de referência. Na análise dos itens, sobressai que 96,86\% dos usuários afirmaram a disponibilidade do prontuário no momento da consulta, e $33,73 \%$ responderam que poderiam acessá-lo se quisessem. Esses resultados da microrregião de Alfenas (MG) foram inferiores aos apresentados por outros estudos (PEREIRA ET AL., 2011; SALA ET AL., 2011; VIANA, 2012).

$\mathrm{O}$ discreto percentual de usuários que demonstraram conhecer o direito de consulta ao prontuário revela que as equipes da ESF dessa microrregião têm um ponto importante a ser desenvolvido na educação em saúde: os direitos dos usuários dos serviços de saúde.

Os atributos Integralidade - Serviços Disponíveis e Integralidade - Serviços Prestados alcançaram baixos escores médios na avaliação dos usuários da APS na microrregião de Alfenas. Esses atributos receberam altos escores dos usuários na maioria das investigações consultadas na literatura (ELIAS ET AL., 2006; MACINKO; ALMEIDA; DE SÁ, 2007; PEREIRA ET AL., 2011; SALA ET AL., 2011; VAN StRALEN ET AL., 2008; VIANA ET AL., 2008). O detalhamento do resultado da Integralidade - Serviços Disponíveis está exposto na tabela 4. 
Tabela 4. Distribuição percentual das respostas dos usuários adultos da Estratégia Saúde da Família aos itens que compõem o atributo Integralidade Serviços Disponíveis, microrregião de Alfenas - MG, 2012. Alfa de Cronbach = 0,807, N=460

\begin{tabular}{|c|c|c|c|c|c|c|c|c|c|c|c|}
\hline \multirow{2}{*}{ Avaliação } & G1 & G2 & G3 & G4 & G5 & G6 & G7 & G8 & G9 & G10 & G11 \\
\hline & $\%$ & $\%$ & $\%$ & $\%$ & $\%$ & $\%$ & $\%$ & $\%$ & $\%$ & $\%$ & $\%$ \\
\hline Com certeza, sim & 45,77 & 32,75 & 25,81 & 74,78 & 61,82 & 62,17 & 53,28 & 23,26 & 26,09 & 6,75 & 25,05 \\
\hline Provavelmente sim & 20,17 & 15,40 & 13,23 & 4,13 & 7,38 & 9,13 & 17,69 & 16,09 & 20,65 & 3,92 & 20,26 \\
\hline $\begin{array}{l}\text { Provavelmente não/não } \\
\text { sei/não lembro }\end{array}$ & 13,88 & 27,11 & 31,02 & 9,13 & 9,76 & 9,78 & 21,40 & 41,30 & 35,87 & 24,18 & 35,51 \\
\hline Com certeza, não & 20,17 & 24,73 & 29,93 & 11,96 & 21,04 & 18,91 & 7,64 & 19,35 & 17,39 & 65,14 & 19,17 \\
\hline Total & 100 & 100 & 100 & 100 & 100 & 100 & 100 & 100 & 100 & 100 & 100 \\
\hline \multirow[b]{2}{*}{ Avaliação } & G12 & G13 & G14 & G15 & G16 & G17 & G18 & G19 & G20 & G21 & G22 \\
\hline & $\%$ & $\%$ & $\%$ & $\%$ & $\%$ & $\%$ & $\%$ & $\%$ & $\%$ & $\%$ & $\%$ \\
\hline Com certeza, sim & 13,02 & 12,58 & 6,74 & 4,12 & 83,70 & 38,70 & 66,45 & 5,68 & 36,18 & 54,45 & 35,29 \\
\hline Provavelmente sim & 18,00 & 16,05 & 2,61 & 1,30 & 7,17 & 24,78 & 10,24 & 2,18 & 17,98 & 18,22 & 18,08 \\
\hline $\begin{array}{l}\text { Provavelmente não/não } \\
\text { sei/não lembro }\end{array}$ & 28,63 & 26,46 & 25,65 & 23,21 & 6,30 & 21,96 & 13,29 & 27,51 & 22,37 & 13,45 & 29,41 \\
\hline Com certeza, não & 40,35 & 44,90 & 65,00 & 71,37 & 2,83 & 14,57 & 10,02 & 64,63 & 23,46 & 13,88 & 17,21 \\
\hline Total & 100 & 100 & 100 & 100 & 100 & 100 & 100 & 100 & 100 & 100 & 100 \\
\hline
\end{tabular}

Fonte: Elaboração própria

Notas: A seguir, apresentamos uma lista de serviços/orientações que você e sua família ou as pessoas que utilizam esse serviço podem necessitar em algum momento. Indique, por favor, se no 'nome do serviço de saúde/ou nome médico/enfermeiro' esses serviços ou orientações estão disponíveis: G1 - Respostas a perguntas sobre nutrição ou dieta. G2 - Verificar se sua família pode participar de algum programa de assistência social ou benefícios sociais. G3 - Programa de suplementação nutricional (ex: leite, alimentos). G4 - Vacinas (imunizações). G5 - Avaliação da saúde bucal (Exame dentário). G6 - Tratamento dentário. G7 - Planejamento familiar ou métodos anticoncepcionais. G8 - Aconselhamento ou tratamento para o uso prejudicial de drogas (lícitas ou ilícitas, ex: álcool, cocaína, remédios para dormir). G9 - Aconselhamento para problemas de saúde mental. G10 - Sutura de um corte que necessite de pontos. G11 - Aconselhamento e solicitação de teste anti-HIV. G12 - Identificação (Algum tipo de avaliação) de problemas auditivos (para escutar). G13 - Identificação (Algum tipo de avaliação) de problemas visuais (para enxergar). G14 - Colocação de tala (ex: para tornozelo torcido). G15 - Remoção de verrugas. G16 - Exame preventivo para câncer de colo de útero (Teste Papanicolau). G17 - Aconselhamento sobre como parar de fumar. G18 - Cuidados pré-natais. G19 - Remoção de unha encravada. G20 - Aconselhamento sobre as mudanças que acontecem com o envelhecimento (ex.: diminuição da memória, risco de cair). G21 - Orientações sobre cuidados no domicílio para alguém da sua família, como: curativos, troca de sondas, banho na cama. G22 - Orientações sobre o que fazer caso alguém de sua família fique incapacitado e não possa tomar decisões sobre sua saúde (ex.: doação de órgãos caso alguém de sua família fique incapacitado para decidir, por exemplo, em estado de coma).

Para essa avaliação do atributo, não é necessário que o usuário tenha recebido os serviços, podendo ser algum familiar, mas o entrevistado deve, sim, saber ou não sobre sua disponibilidade (BRASIL, 2010). Distinguem-se os itens que foram computados com elevados percentuais positivos pelos usuários. São eles: o exame de papanicolau, a imunização, o pré-natal e as orientações sobre cuidados no domicílio. Resultados com altos percentuais de avaliação positiva para esses itens também foram encontrados em outras avaliações da ESF (PEREIRA ET AL., 2011; SALA ET AL., 2011; VIANA, 2012).

Em contrapartida, destacam-se os baixos percentuais para os procedimentos considerados cirúrgicos e invasivos, o aconselhamento para problemas de saúde mental, o aconselhamento/tratamento da dependência do uso de drogas lícitas e ilícitas e do tabaco. Outras avaliações tiveram resultados semelhantes (PEREIRA ET AL., 2011; SALA ET AL., 2011; VIANA, 2012). O não reconhecimento da ESF 
como uma porta de entrada para o atendimento dessas demandas é uma lacuna importante em sua atuação - ao se considerar a importância epidemiológica desses temas, o impacto sobre as famílias e o custo para a sociedade - por seus desdobramentos, tais como violência urbana, violência doméstica e incapacitação para o trabalho.

Os profissionais que atuam na ABS carregam a responsabilidade de lidar com uma clientela portadora de uma diversidade de sofrimentos, entre eles, os psíquicos. A estrutura atual do SUS ainda é insuficiente na oferta de respostas a ocorrências de violência e necessita ampliar o olhar sobre a diversidade de situações que permeiam o processo saúde-doença. (PEREIRA ET AL., 2011, P.52).

O delineamento do resultado do atributo Integralidade - Serviços Prestados é abordado na tabela 5 .

Tabela 5. Distribuição percentual das respostas dos usuários adultos da Estratégia Saúde da Família aos itens que compõem o atributo Integralidade Serviços Prestados, microrregião de Alfenas - MG. Alfa de Cronbach = 0,882, H1 a H11 N=508; H12 e H13 N = 438)

\begin{tabular}{|c|c|c|c|c|c|c|c|c|c|c|c|c|c|}
\hline \multirow{2}{*}{ Avaliação } & H1 & $\mathrm{H} 2$ & H3 & H4 & H5 & $\mathrm{H} 6$ & $\mathrm{H7}$ & $\mathrm{H} 8$ & H9 & $\mathrm{H} 10$ & H11 & $\mathrm{H} 12$ & $\mathrm{H} 13$ \\
\hline & $\%$ & $\%$ & $\%$ & $\%$ & $\%$ & $\%$ & $\%$ & $\%$ & $\%$ & $\%$ & $\%$ & & \\
\hline Com certeza, sim & 59,14 & 42,24 & 18,11 & 21,02 & 60,90 & 68,84 & 62,13 & 16,50 & 4,72 & 23,23 & 30,18 & 40,63 & 40,32 \\
\hline Provavelmente sim & 12,18 & 13,36 & 5,91 & 12,38 & 12,18 & 8,68 & 16,37 & 8,25 & 2,56 & 14,17 & 14,20 & 11,74 & 14,41 \\
\hline $\begin{array}{l}\text { Provavelmente não/ } \\
\text { não sei/não lembro }\end{array}$ & 8,64 & 11,59 & 25,79 & 22,20 & 7,86 & 7,10 & 6,11 & 23,18 & 11,81 & 12,01 & 9,27 & 8,58 & 8,33 \\
\hline Com certeza, não & 20,04 & 32,81 & 50,20 & 44,40 & 19,06 & 15,38 & 15,38 & 52,06 & 80,91 & 50,59 & 46,35 & 39,05 & 36,94 \\
\hline Total & 100 & 100 & 100 & 100 & 100 & 100 & 100 & 100 & 100 & 100 & 100 & 100 & 100 \\
\hline
\end{tabular}

Fonte: Elaboração própria

Notas: A seguir, apresentamos uma lista de serviços/orientações que você e sua família ou as pessoas que utilizam esse serviço podem necessitar em algum momento. Indique, por favor, se no 'nome do serviço de saúde/ou nome médico/enfermeiro' esses serviços ou orientações estão disponíveis: H1- Conselhos sobre alimentação saudável ou sobre dormir suficientemente. H2 - Segurança no lar, como guardar medicamentos em segurança. H3 - Aconselhamento sobre o uso de cinto de segurança ou assentos seguros para crianças ao andar de carro. H4 - Maneiras de lidar com conflitos de família que podem surgir de vez em quando. H5 - Conselhos a respeito de exercícios físicos apropriados para você. H6 - Testes de sangue para verificar os níveis de colesterol. H7 - Verificar e discutir os medicamentos que você está tomando. H8 - Possíveis exposições a substâncias perigosas (ex: veneno para formiga/para rato, água sanitária), no seu lar, no trabalho ou na sua vizinhança. H9 - Perguntar se você tem uma arma de fogo e orientar sobre como guardá-la com segurança. H10 - Como prevenir queimaduras (ex: causadas por água quente, óleo quente, outras substâncias). H11 Como prevenir quedas. H12 - Só para mulheres: como prevenir osteoporose ou ossos frágeis. H13 - Só para mulheres: o cuidado de problemas comuns da menstruação ou menopausa.

Nesse ponto, a avaliação é voltada para as ações e para os serviços utilizados pelo usuário ou por alguém de sua família, na unidade da ESF. A maioria dos itens desse atributo recebeu avaliações negativas, com destaque para os menores índices relacionados à segurança no acondicionamento de arma de fogo; ao uso do cinto de segurança e de dispositivo de retenção para crianças; ao risco de exposição a substâncias tóxicas em casa ou no trabalho; ao aconselhamento sobre conflitos familiares; e à prevenção de queimaduras. Nessa microrregião, a quarta maior causa de mortalidade hospitalar acontece por causas externas, que englobam morte por acidentes no transporte, homicídios e suicídios (BRASIL, 2013). O resultado negativo desses itens que demonstram fragilidades para se atingir a integralidade coincide com os achados de Viana (2012), Sala 
et al. (2011) e Pereira et al. (2011). Referindo-se às ações educativas e de aconselhamento, Pereira et al. (2011) dizem que as ações relativas à prevenção de doença e à promoção da saúde ainda são incipientes, revelando a manutenção do modelo assistencial centrado em práticas curativas.

Os baixos escores dos dois atributos que aferiram a efetivação da Integralidade contrariam a afirmação de Sala et al. (2011), de que o modelo de organização da APS nos moldes da ESF apresenta, em tese, melhores características para a efetivação dos princípios da integralidade, uma vez que as ações são realizadas em um território estruturado, permitindo o planejamento das ações a partir das condições de vida e de saúde da população adscrita, e não a partir da demanda por assistência.

Essa avaliação ruim pode ser interpretada de duas formas: a primeira é que, embora o instrumento tenha sido validado no Brasil, o rol de serviços elencados não está adequado à realidade das ESF participantes do estudo. A segunda é que as ações e os serviços propostos estão aquém das necessidades dos usuários e não contemplam todo o espectro da atuação da APS, ficando as práticas de prevenção e de promoção subjugadas às práticas tradicionais de tratamento e de reabilitação. Outrossim, para a população que reside na microrregião de Alfenas, o modelo de organização da APS, nos moldes da ESF, não se mostrou comprometido com a integralidade e com a clínica ampliada.

A avaliação da Orientação Familiar recebeu um baixo escore médio por parte dos entrevistados, distinguindo-se a avaliação positiva para o item que se refere ao questionamento do profissional de saúde sobre doenças que já ocorreram na família. Os resultados negativos para esse atributo foram uma unanimidade nos estudos realizados no Brasil, que utilizaram o PCATool, independentemente da versão proposta (ELIAS ET AL., 2006; IBAÑEZ ET AL., 2006; MACINKO; ALMEIDA; DE SÁ, 2007; OLIVEIRA, 2007; OLIVEIRA ET AL., 2013; PEREIRA ET AL.
2011; SALA ET AL., 2011; VAN STRALEN ET AL., 2008; VIANA ET AL., 2008; VIANA, 2012). As piores avaliações foram sobre a participação do usuário no tratamento $(40,12 \%)$, assim como em outros estudos (PEREIRA ET AL., 2011; SALA ET AL., 2011; VIANA, 2012).

Considera-se, assim, que o usuário não experimenta/percebe a qualidade de Orientação Familiar nas ações dos serviços de saúde. Mais importante do que verificar o não alcance de um índice numérico que meça a qualidade assistencial é refletir sobre a persistência da prática centrada no profissional, oposto do que foi proposto na mudança de modelo assistencial a ser efetivada pelo SUS, a partir da reorganização da APS com a ESF, para a qual a unidade de cuidado mais importante é a família, expressa na própria denominação da mesma.

O atributo Orientação Comunitária recebeu avaliação considerada de baixo escore, assim como em outros trabalhos realizados com usuários sobre a assistência com adultos (ELIAS ET AL., 2006; IBAÑEZ ET AL., 2006; OLIVEIRA, 2007; PEREIRA ET AL., 2011; SALA ET AL., 2011; VIANA ET AL., 2008), com mulheres (VIANA, 2012) e com idosos (OLIVEIRA ET AL., 2013). O estudo de Macinko, Almeida e Sá (2007) obteve avaliação positiva dos usuários para esse atributo da APS.

Elias et al. (2006) chamam a atenção para o fato de um estudo de avaliação encontrar um valor baixo para o atributo Orientação Comunitária, pois é um dos componentes essenciais da ESF. Para esses autores, o resultado esperado seria uma boa avaliação.

Nesse atributo, os itens que tratam das visitas domiciliares (87,37\%) e do reconhecimento dos problemas de saúde da comunidade pelos profissionais do serviço de saúde (74,37\%) receberam avaliações positivas. A visita domiciliar talvez seja uma das ações que têm maior visibilidade e impacto junto à população, já que faz parte da rotina das atividades dos membros da ESF, sobretudo dos ACS.

Os próximos itens não atingiram um percentual alto de avaliações positivas. Tiveram como objetivo analisar se o serviço de saúde 
ouve opiniões e ideias da comunidade sobre como melhorar os serviços de saúde $(53,39 \%)$; se afere a satisfação da população sobre os serviços ofertados $(42,14 \%)$; se pesquisa sobre os problemas de saúde da comunidade $(41,94 \%)$; e de que forma estimula a participação da mesma no controle social (27,48\%). Os estudos de Viana (2012), Pereira et al. (2011) e Sala et al. (2011) também encontraram baixos percentuais para esses itens do atributo Orientação Comunitária. A população reconhece que os profissionais identificam quais são os problemas das comunidades, mas também enxergam que o serviço não a envolve no planejamento, no acompanhamento e na avaliação do cuidado desenvolvido. Sobre esse atributo, concorda-se com Pereira et al. (2011) na reflexão de que esse resultado negativo indica a necessidade de uma revisão do processo de trabalho, no qual aquele que utiliza o serviço tenha efetivamente participação na formulação de propostas e intervenções.

O Escore Essencial e o Escore Geral obtiveram valores médios baixos, revelando que a prática da ESF na microrregião de Alfenas não está orientada pelos atributos da APS. Alguns estudos não realizaram essa aferição (IBAÑEZ ET AL., 2006; PEREIRA ET AL., 2011; SALA ET AL., 2011). Os que realizaram atingiram valores baixos para o Escore Geral e para o Essencial (OLIVEIRA, 2007; OLIVEIRA ET AL., 2013; VIANA, 2012). NoS estudos que realizaram o chamado Índice da Atenção Básica, que consiste em realizar média única dos escores, correspondente ao Escore Geral, os resultados foram altos (ELIAS ET AL., 2006; MACINKO; ALMEIDA; DE SÁ, 2007; VAN STRALEN ET AL., 2008; VIANA ET AL., 2008).

Neste estudo, os atributos que tiveram um desempenho ruim guardam relação entre si. Os atributos Orientação Familiar e Comunitária acontecem quando o alcance da Integralidade fornece uma base para a consideração dos pacientes dentro de seus ambientes; quando a avaliação das necessidades para a atenção integral considera o contexto social e familiar e sua exposição a ameaças à saúde; e quando o desafio da Coordenação da Atenção se defronta com recursos familiares limitados (STARFIELD, 2002).

\section{Considerações finais}

Os usuários da ESF na microrregião de Alfenas têm-na como fonte regular de atenção às suas necessidades de saúde ao longo da vida, mas encontram dificuldades no acesso devido às várias barreiras organizacionais, sobretudo nas formas de comunicação e nos horários de funcionamento das mesmas. A estrutura e o processo de trabalho favorecem os atendimentos de rotina durante a semana, nos quais é imprescindível a presença da pessoa para informação, para agendamento e para atendimento. Esses resultados levam a inferir que, a despeito da alta cobertura dos serviços da ESF na microrregião de Alfenas, a mesma não se constitui numa porta de entrada abrangente para o SUS. A posição da ESF como centro coordenador do cuidado nos demais níveis assistenciais é reconhecida. No entanto, a avaliação indica que esse atributo ainda necessita de aperfeiçoamentos, sobretudo na contrarreferência.

Parte dos usuários percebe a verticalização das práticas de saúde e vivência um vínculo fraco com os serviços, fruto de práticas em saúde que não atingiram o (re)conhecimento do usuário como pessoa, como parte de uma família e como membro de uma comunidade, que tem uma opinião a ser considerada na organização das ações e dos serviços para que as necessidades da localidade na qual a ESF está inserida sejam atendidas.

Os serviços reconhecidos como disponíveis expõem uma prática bastante tradicional nas unidades da ESF da microrregião de Alfenas, com ações preventivas consolidadas, centradas na saúde da mulher e da criança, e que não revelam espaço para a promoção da saúde e para a diversidade das necessidades da população atendida. 
Denota-se a necessidade de se orientar as práticas de acordo com os perfis social, econômico e epidemiológico da comunidade na qual a ESF está inserida, por meio da realização do diagnóstico local, elaborado com consulta à comunidade e aos bancos de dados oficiais, na tentativa de superar a verticalização das ações e dos serviços de saúde. No avanço dessa prática, também é possível oferecer subsídios para a organização e para o aprimoramento da rede de serviços de saúde nos demais níveis assistenciais. Demanda-se o emprego de ferramentas de abordagem familiar e comunitária, o aperfeiçoamento da forma de comunicação e o relacionamento entre profissional, serviço e usuários, além da sensibilização de todos, a fim de suprir a lacuna da participação popular e do controle social evidenciado.

Obviamente, não é possível que a APS responda a todas as demandas de saúde da população de uma região característica de um país em desenvolvimento, mas o caráter técnico da APS necessita constantemente de aperfeiçoamento. Esta pesquisa contribui no sentido de que, ao se avaliar, indica-se, pontualmente, onde é preciso avançar para o alcance de uma assistência integral e de qualidade. A partir dessas constatações, é também preciso militar para que as políticas se instaurem, concretizem-se.

\section{Referências}

\author{
ANDRADE, L. O. M. DE; BUENO, I. C. DE H. C.; \\ BEZERRA, R. C. Atenção Primária à Saúde e Estratégia \\ Saúde da Família. In: CAMPOS GWS, MINAYO MCS, \\ AKERMAN M, DRUMOND JUNIOR M, C. Y. (Ed.). \\ Tratado de Saúde Coletiva. São Paulo: Hucitec/Fiocruz, \\ 2006, p. 783-837.
}

BODSTEIN, R. et al. Estudos de Linha de Base do Projeto de Expansão e Consolidação do Saúde da Família (ELB/Proesf): considerações sobre seu acompanhamento. Ciência \& Saúde Coletiva, Rio de Janeiro, v. 11, n. 3, p. 725-731, set. 2006.

BRASIL. MINISTÉRIO DA SAÚDE. Mortalidade Brasil. Disponível em: <http://tabnet.datasus.gov.br/ cgi/deftohtm.exe?sim/cnv/obtlouf.def>. Acesso em: 22 jul. 2013.

Ministério da saúde. Secretaria de Atenção em Saúde. Departamento de Atenção Básica. Manual do instrumento de avaliação da atenção primária à saúde: Primary Care Assessment Tool PCATool - Brasil. Brasília: Ministério da Saúde, 2010, p. 82

DONABEDIAN, A. Evaluating the quality of medical care. 1966. The Milbank quarterly, v. 83, n. 4, p. 691-729, jan. 2005.

ELIAS, P. E. et al. Atenção Básica em Saúde: comparação entre PSF e UBS por estrato de exclusão 
social no município de São Paulo. Ciência \&t Saúde

Coletiva, Rio de Jeniro, v. 11, n. 3, p. 633-641, set. 2006.

GIL, A. C. Métodos e Técnicas de pesquisa social. São

Paulo: Atlas, 1999, p. 206

GIL, C. R. R. Atenção primária, atenção básica e saúde da família: sinergias e singularidades do contexto brasileiro. Cadernos de Saúde Pública, São Paulo, v. 22, n. 6, p. 1171-1181, jun. 2006

GIOVANELLA, L.; ESCOREL, S.; MENDONÇA, M. H. M. Porta de entrada pela atenção básica? Integração do PSF à rede de serviços de saúde. Saúde em Debate, Rio de Janeiro, v. 27, n. 65, p. 278-89, 2003.

IBAÑEZ, N. et al. Avaliação do desempenho da atenção básica no Estado de São Paulo. Ciência \&t Saúde Coletiva, Rio de Janeiro, v. 11, n. 3, p. 683-703, set. 2006.

MACINKO, J.; ALMEIDA, C.; DE SÁ, P. K. A rapid assessment methodology for the evaluation of primary care organization and performance in Brazil. Health policy and planning; London, v. 22, n. 3, p. 167-77, maio 2007.

MACINKO, J.; ALMEIDA, C.; OLIVEIRA, E. Avaliação das características organizacionais dos serviços de atenção básica em Petrópolis: teste de uma metodologia. Saúde em Debate, Rio de Janeiro, v. 27, n. 65, p. 243-256, 2003.

NOVAES, H. M. D. Avaliação de programas, serviços e tecnologias em saúde. Revista Saúde Pública, São Paulo, v. 34, n. 5, p. 547-549, out. 2000.

OLIVEIRA, M. M. C. DE. Presença e Extensão dos Atributos da Atenção Primária entre os Serviços da Atenção Primária em Porto Alegre: uma análise agregada. [s.l.] Universidade Federal do Rio Grande do Sul, 2007.

OLIVEIRA, E. B. et al. Avaliação da qualidade do cuidado a idosos nos serviços da rede pública de atenção primária à saúde de Porto Alegre, Brasil. Rev. Bras. Med. Fam. Comunidade, Rio de Janeiro, v. 8, n. 29, p. 264-273, 2013.
PEREIRA, M. J. B. et al. Avaliação das características organizacionais e de desempenho de uma unidade de Atenção Básica à Saúde. Revista Gaúcha de Enfermagem, Porto Alegre, v. 32, n. 1, p. 48-55, mar. 2011.

POLIT, D.; BECK, C.; HUNGLER, B. Fundamentos de pesquisa em enfermagem. 5. ed. Porto Alegre: Artmed, 2004, p. 487

REIS, R. S. et al. Acesso e utilização dos serviços na Estratégia Saúde da Família na perspectiva dos gestores, profissionais e usuários. Ciência \& Saúde Coletiva, Rio de Janeiro, v. 18, n. 11, p. 3321-3331, nov. 2013.

SALA, A. et al. Integralidade e Atenção Primária à Saúde: avaliação na perspectiva dos usuários de unidades de saúde do município de São Paulo. Saúde e Sociedade, São Paulo, v. 20, n. 4, p. 948-960, dez. 2011.

STARFIELD, B. Atenção primária: equilíbrio entre necessidades de saúde, serviços e tecnologia. Brasília: Unesco, 2002, p. 726

VAN STRALEN, C. J. et al. Percepção dos usuários e profissionais de saúde sobre atenção básica: comparação entre unidades com e sem saúde da família na Região Centro-Oeste do Brasil. Cadernos de Saúde Pública, São Paulo, v. 24, n. supl. 1, p. 148-158, 2008.

VIANA, A. L. D’ÁVILA et al. Atenção básica e dinâmica urbana nos grandes municípios paulistas, Brasil. Cadernos de Saúde Pública, São Paulo, v. 24, n. supl. 1, p. s79-s90, 2008.

VIANA, L. M. M. Avaliação da atenção primária à saúde de Teresina na perspectiva das usuárias. [s.l.] Universidade Federal do Piauí, 2012.

Recebido para publicação em março de 2014

Versão final em setembro de 2014

Conflito de interesses: inexistente

Suporte financeiro: CNPq, processo número 473768/2011-9 